\title{
Analysis of the lipids mobilized by adipokinetic hormones in the firebug Pyrrhocoris apterus (Heteroptera: Pyrrhocoridae)
}

\author{
Iva BÁRTU゚ ${ }^{1,2}$, Aleš TOMČALA ${ }^{1,3}$, RAdomír SOCHA ${ }^{1}$, Petr ŠIMEK ${ }^{1}$ and DALIBOR KODRÍK ${ }^{1,2 *}$ \\ ${ }^{1}$ Institute of Entomology, Biology Centre, Academy of Sciences, Branišovská 31, 37005 České Budějovice, Czech Republic; \\ e-mail: kodrik@entu.cas.cz \\ ${ }^{2}$ Faculty of Science, University of South Bohemia, Branišovská 31, 37005 České Budějovice, Czech Republic \\ ${ }^{3}$ Institute of Organic Chemistry and Biochemistry, Academy of Sciences, Flemingovo náměstí 2, 16610 Praha 6, Czech Republic
}

Key words. Heteroptera, Pyrrhocoris apterus, AKH, lipid, triacylglycerol, diacylglycerol, fatty acid, haemolymph, fat body, linden seed, HPLC, ESI-MS, mass spectrometry

\begin{abstract}
Effects of two adipokinetic hormones (Pyrap-AKH and Peram-CAH-II) on the presence of diacylglycerol (DG) molecular species and their fatty acid (FA) constituents in the haemolymph of the firebug Pyrrhocoris apterus were investigated using liquid chromatography (HPLC) and electrospray ionization mass spectrometry (ESI-MS). The results show that DGs with characteristic FAs are preferentially mobilized from the fat body (FB) by the action of both the AKHs produced by P. apterus. Both the macropterous and brachypterous morphs have similar DG and FA profiles. A difference in the action of the Pyrap-AKH and the PeramCAH-II, however, results in distinct differences in the distribution of FAs in the macropterous morph. It seems that C16 to a slight extent and unsaturated C18 FAs mainly play a dominant role in the AKH based action, in particular linoleic acid (18:2), which represents $50-60 \%$ of the total DG mobilized. The metabolically active C16 and C18 FAs are preferentially absorbed from the linden seeds and accumulated in the FB. The relationships between AKH action and FA distribution in DGs in P. apterus, compared to other insect species are summarized and discussed in detail.
\end{abstract}

\section{INTRODUCTION}

In insects, storage lipids, triacylglycerols (TGs), occur mainly in the fat body (FB), an organ analogous to vertebrate adipose tissue and liver, where they make up about 90\% of the total lipids (Arrese \& Wells, 1994; Canavoso et al., 1998, 2001; Arrese \& Soulages, 2010). However the major class of lipids in the haemolymph are diacylglycerols (DGs) that are predominantly bound to carrier protein lipophorins (Chino \& Downer, 1982). Unlike in vertebrates, where free fatty acids (FAs) are released into the bloodstream and transported bound to albumin, the DGs serve as the major transport lipid shuttle in insect haemolymph, of which more than $90 \%$ are $s n$-1,2-isomers (Van der Horst, 1982; Arrese et al., 1996).

The DG molecule is transported from the FB using a lipid transport particle (Van Heusden \& Law, 1989) to high-density lipophorin (HDLp) in the haemolymph. HDLp stability and capacity is increased by apolipoprotein-III (apoLp-III) and the mass of the resulting particle, called a low-density lipophorin (LDLp), is about twice that of HDLp. The LDLp transports the DGs to target tissues (mostly muscles) where the DGs are hydrolyzed by lipophorin lipase to free FAs and glycerol. Then, the apoLp-III dissociates from LDLp and is converted to HDLp, which returns to the FB to transport the next DG molecule (Canavoso et al., 2001; Van der Horst \& Ryan, 2005).

\footnotetext{
* Corresponding author.
}

The DG molecular species differ in their constituent FAs. Several dozen FAs have been detected in insect haemolymph, but most are 8-9 FAs with 12-18 carbon atoms and include the saturated FAs: lauric (C12:0), myristic (C14:0), palmitic (C16:0) and stearic (C18:0), the monounsaturated FAs: myristoleic (C14:1), palmitoleic $(\mathrm{C} 16: 1)$ and oleic $(\mathrm{C} 18: 1)$, and polyunsaturated FAs: linoleic (C18:2) and linolenic (C18:3). The monounsaturated FAs consist primarily of the cis- $\Delta-9$-isomer and the $C 18$ polyunsaturated FAs the cis- $\Delta-9-12-$ and cis- $\Delta-9$ 12-15 isomers (Downer, 1985; Schneider \& Dorn, 1994).

Insect energy metabolism, predominantly activation of lipids, is controlled by adipokinetic hormones (AKHs). The latter are a large group of neuropeptides consisting of 8-10 amino acids, which are synthesized and released from the corpora cardiaca, neuroendocrine glands connected to the brain (Gäde et al., 1997). Generally, AKHs behave as typical stress hormones by stimulating catabolic reactions, which make energy more available, while inhibiting synthetic reactions. Their crucial role in the mobilization of lipids is well known (e.g. review articles - Canavoso et al., 2001; Gäde \& Goldsworthy, 2003; Van der Horst \& Ryan, 2005), but they are also involved in a number of accompanying processes (Kodrík, 2008). Currently, almost 50 different AKHs have been characterized and their physiological effects investigated in insects belonging to various insect orders (Gäde et al., 1997; Gäde, 2009). The AKH induced mobilization of lipids from the FB was first reported for the locust, Locusta 
migratoria, in which they fuel long-distance flights (Mayer \& Candy, 1969). This species is the classical insect model with three AKHs: Locmi-AKH-I, -II and -III, of which AKH-I is the most effective in mobilizing lipids (Goldsworthy, 1994). In Heteroptera, at least three species have more than one AKH peptide, namely two species in the family Pyrrhocoridae (Pyrrhocoris apterus and Dysdercus intermedius with Pyrap-AKH and PeramCAH-II) (Kodrík et al., 2000, 2002) and one in the family Tessaratomidae (Encosternum delegorguei with PanboRPCH and Schgr-AKH-II) (Kodrík et al., 2010).

Although there is a lot of information on the physiological, biochemical and metabolic roles of AKHs in insects, little is known about the effects of individual AKHs on the mobilization of particular DG molecular species in insect species with more than one AKH. The DGs and/or FAs are not uniformly mobilized from the FB of L. migratoria after injection with AKHs. The individual DG species were mobilized selectively: the DGs containing $\mathrm{C} 18$ and $\mathrm{C} 16 \mathrm{FAs}$ and especially the $\mathrm{C} 18$ unsaturated oleic acid were preferred (Tomčala et al., 2010). In addition Locmi-AKH-I preferred mobilization of unsaturated FAs while Locmi-AKH-II and -III mobilization of saturated FAs.

A question arises whether the above results are specific for L. migratoria or whether AKHs might also selectively mobilize lipids in other species possessing more than one AKH. $P$. apterus is a suitable insect model for this kind of research because it has two AKHs (Pyrap-AKH and Peram-CAH-II) (Kodrík et al., 2000, 2002) and uses lipids to fuel its locomotion (Socha \& Kodrík, 1999; Socha et al., 1999). Moreover, this bug is characterized by a conspicuous wing polymorphism, and the macropterous (long-winged) and brachypterous (short winged) morphs (Socha, 1993) differ in their life history strategies (Socha \& Zemek, 2003; Socha et al., 2005; Socha \& Sula, 2008).

The aim of the present study was to determine whether (1) the DGs and FAs are selectively mobilized by Pyrap-AKH and Peram-CAH-II in P. apterus, (2) the spectrum of lipids present in the bugs differs from that found in their food (linden seeds), and (3) the mobilization of lipids in the adults of the two wing morphs differ. The results might contribute to extension of our knowledge why certain insect species possess more than one AKH.

\section{MATERIAL AND MEHODS}

\section{Experimental animals}

The stock cultures of P. apterus used in the present study, originated from specimens collected at České Budějovice (Czech Republic, $49^{\circ} \mathrm{N}$ ) (Socha \& Šula, 1996). All stages from egg to adult were kept in small glass jars with an ad libitum supply of linden seeds (Tilia cordata) and water, and kept at a constant temperature of $26 \pm 1^{\circ} \mathrm{C}$ and under long-day conditions (18L : 6D). Two different groups of 10-day old adult females (for reasons see Socha \& Kodrík, 1999) were used in the experiments: reproductive brachypterous and macropterous individuals with a non-diapause type of reproductive arrest.

\section{Chemicals}

The $P$. apterus adipokinetic hormones Pyrap-AKH (pGluLeu-Asn-Phe-Thr-Pro-Asn-Trp- $\mathrm{NH}_{2}$ ) (Kodrík et al., 2000) and Peram-CAH-II (pGlu-Leu-Thr-Phe-Thr-Pro-Asn-Trp- $\mathrm{NH}_{2}$ ) (Kodrík et al., 2002) were commercially synthesised by Dr. Lepša, the Vidia Company (Praha, Czech Republic). TG and DG standards and other chemicals used in the experiments, including HPLC and ESI-MS analyses, were purchased from Sigma-Aldrich (Praha, Czech Republic).

\section{Hormonal treatment and preparation of samples}

A dose of 10 pmol Pyrap-AKH or Peram-CAH-II dissolved in $2 \mu 120 \%$ methanol in Ringer saline (Kodrík et al., 2000) was injected through the metathoracic-abdominal intersegmental membrane into the thorax of the experimental bugs. Control bugs were injected with $2 \mu 1$ of solvent. A haemolymph sample was taken from each of the 20 females just before and $90 \mathrm{~min}$ after they were injected. Haemocytes were removed from the samples by centrifuging at $13,000 \mathrm{~g}$ for $2 \mathrm{~min}$ at $4{ }^{\circ} \mathrm{C}$. FBs were dissected from bugs by opening the body from ventral side. The lipids in $10 \mu$ l of the pooled haemolymph samples $(\mathrm{n}=3$; each $\underline{\mathrm{n}}$ from 20 bugs) and weighed samples of FB (usually 10 $\mathrm{mg} /$ analysis, $\mathrm{n}=12$ ) were extracted immediately after dissection using a chloroform : methanol (ratio - 2:1) solution following the method of Folch et al. (1957) as modified by Košt'ál \& Šimek (1998).

The same method was used to extract lipids from a sample of the linden seeds that were used to feed the bugs. The testa of each of the seeds was removed and the content of the seeds weighed, homogenised and extracted using the same chloroform : methanol solution as mentioned above $(n=7)$.

\section{HPLC and ESI-MS analyses}

High performance liquid chromatography (HPLC) combined with electrospray ionization mass spectrometry (ESI-MS) was done using a quadrupole ion trap LCQ mass spectrometer (Thermo, San Jose, CA, USA) coupled to a Rheos 2000 ternary HPLC system (Flux, Basel, Switzerland), equipped with a FAMOS autosampler and Thermos thermostat (both LC Packings-Dionex, Amsterdam, The Netherlands) as described in Tomčala et al. (2006). The stored dry samples were dissolved in $1 \mathrm{ml}$ methanol and $5 \mu \mathrm{l}$ aliquots were injected into a $150 \times 2.0$ $\mathrm{mm}$ i.d. $3 \mu \mathrm{m}$ Gemini HPLC column (Phenomenex, Torrance, CA, USA). The mobile phase was composed of (A) $5 \mathrm{mmol} \mathrm{l}^{-1}$ ammonium acetate in methanol, (B) water and (C) isopropanol. A linear gradient of $\mathrm{A}: \mathrm{B}: \mathrm{C}$ changing from $92: 8: 0$ to $50: 0: 50$ within $37 \mathrm{~min}$ was used with a flow rate of $150 \mu \mathrm{min}^{-1}$. The column temperature was maintained at $30^{\circ} \mathrm{C}$. The mass spectrometer was operated in the positive ion detection mode at +4 $\mathrm{kV}$ with a capillary temperature of $220^{\circ} \mathrm{C}$. Nitrogen was used as a shielding and auxiliary gas. Mass range of 440-1100 Da were scanned every $0.5 \mathrm{~s}$ to obtain the ESI mass spectra of the respective DGs and TGs. For the investigation of the DG and TG structures the CID (collision induced dissociation) multi-stage ion trap tandem mass spectra $\mathrm{MS}^{2}$ were recorded, respectively, at 5 and $3 \mathrm{Da}$ isolation windows. Maximum ion injection time was $100 \mathrm{~ms}$ and the normalized collision energy was set to $30 \%$ for the CID $\mathrm{MS}^{2}$ scan type. The ammonium adducts of molecules, $\left[\mathrm{M}+\mathrm{NH}_{4}\right]^{+}$obtained by positive ESI were used for determining the molecular weight of each DG or TG molecular species. The number of carbon and double bonds can be calculated from the molecular weight of each particular DG or TG entity. The CID of $\left[\mathrm{M}+\mathrm{NH}_{4}\right]^{+}$ions results in neutral losses of $\mathrm{NH}_{3}$ (i.e. molecular ion $[\mathrm{M}+\mathrm{H}]^{+}$is observed) and acyl side-chain (as a carboxylic acid [M-RCOO $]^{+}$) in the generation of the monoacyl product ion. This fragmentation is characteristic of all 
molecular species of DGs and TGs. For example two DGs with $\mathrm{m} / \mathrm{z} 638\left(\left[\mathrm{M}+\mathrm{NH}_{4}\right]^{+}-\mathrm{DG}\right.$ of $\left.\mathrm{mw} 620 \mathrm{Da}\right)$ were present in all samples. Inspection of the CID $\mathrm{MS}^{2}$ spectra revealed two isomeric DGs, namely DG 18:1/18:1 and DG 18:0/18:2 at $15.7 \mathrm{~min}$ and $16.2 \mathrm{~min}$, respectively. The diagnostic fragment ions in the CID ESI-MS ${ }^{2}$ spectra in the former case are represented by mass $\mathrm{m} / \mathrm{z} 339$ (loss of C18:1) and latter case by masses m/z 337 (loss of C18:0) and m/z 341 (loss of C18:2) (Mu \& Hoy, 2000).

The identification of particular FAs in TG molecules were based on computer-assisted interpretation of $\mathrm{ES} \mathrm{MS}^{2} \mathrm{TG}$ spectra using software TriglyAPCI developed by Cvačka et al. (2006). Although the software is primarily designed for APCI MS spectra, it can be used to determine FAs in TGs obtained using ESI $\mathrm{MS}^{2}$ spectra, because the pattern in the fragmentation of the molecules in both ionisations is very similar.

\section{FA content}

Relative content of FAs in the DGs in the haemolymph and the FB TGs (see Fig. 4) was calculated in two steps: (1) from the theoretical FA composition of particular DG (see Table 1, $2^{\text {nd }}$ column) or TG molecular species (see Table $2,2^{\text {nd }}$ column), and (2) from the relative percentage of DG in the total lipid mobilized (see Fig. 3) or TG in FB (see Table 2, penultimate and ultimate columns)

\section{Data presentation and statistical analysis}

The results were plotted using the graphic program Prism (GraphPad Software, version 5.0, San Diego, CA, USA) (Figs 3, 4 and 6$)$. The bar graphs represent the mean \pm SD. Statistically significant differences at the $5 \%$ level were evaluated using t-tests.

\section{RESULTS}

\section{HPLC/ESI-MS analyses of lipids in} chloroform-methanol extracts of haemolymph

The analyses resulted in the generation of a number of MS peaks (Figs 1A1-C1 and 2A1-C1) from which those corresponding to a set of DG standards, with retention times from 7.5 to $21.5 \mathrm{~min}$ (data not shown), were subjected to further detailed investigation. The DG fractions of molecular mass 500-700 Da in the haemolymph of control brachypterous (Fig. 1A1) and macropterous (Fig. 2A1) bugs, and experimental bugs of both wing morphs injected with 10 pmol Pyrap-AKH (Figs 1B1 and 2B1) or 10 pmol Peram-CAH-II (Figs $1 \mathrm{C} 1$ and 2C1) were studied in detail using corresponding ESI-MS analyses (Fig. 1A2 for analysis of the data of Fig. 1A1, Fig. 1B2 for 1B1, Fig. $1 \mathrm{C} 2$ for $1 \mathrm{C} 1$, and Fig. 2A2 for Fig. 2A1, Fig. 2B2 for $2 \mathrm{~B} 1$ and Fig. $2 \mathrm{C} 2$ for $2 \mathrm{C} 1$ ). These analyses revealed the presence of a relatively large number of DG molecular species, of which about 10 were present only in negligible amounts and had no substantial effect on the total lipid level in the haemolymph. A characteristic set of ten DGs, of which five were present in two different molecular forms are summarized in Table 1. They made up more than $90 \%$ of the total DG in the haemolymph of both the morphs studied and therefore were selected for further study. Their structures were verified by the CID $\mathrm{MS}^{2}$ scan (data not shown) and their molecular masses ranged from 590 to $622 \mathrm{Da}$. The CID $\mathrm{MS}^{2}$ scan revealed the nature of the acyl residues in the DG structure, but not their stereospecific analysis and the 1,2 and 3 positioning of the acyls in the structure. However, based on previous studies
TABLE 1. Characterization of DGs detected in the haemolymph of brachypterous and macropterous bugs injected with Pyrap-AKH and Peram-CAH-II peptides. FAs are described by the following ratio - number of carbon atoms : number of double bonds in the molecule; the DGs existing in two FA variants are labelled (a) or (b).

\begin{tabular}{cc}
\hline DG mw (Da) & Theoretical FA composition \\
\hline 590 & $16: 1 / 18: 2$ \\
592 & $16: 0 / 18: 2$ \\
594 & $16: 0 / 18: 1$ \\
$604(\mathrm{a})$ & $17: 1 / 18: 2$ \\
$604(\mathrm{~b})$ & $17: 0 / 18: 3$ \\
$606(\mathrm{a})$ & $17: 1 / 18: 1$ \\
$606(\mathrm{~b})$ & $17: 0 / 18: 2$ \\
614 & $18: 2 / 18: 3$ \\
$616(\mathrm{a})$ & $18: 2 / 18: 2$ \\
$616(\mathrm{~b})$ & $18: 1 / 18: 3$ \\
$618(\mathrm{a})$ & $18: 1 / 18: 2$ \\
$618(\mathrm{~b})$ & $18: 0 / 18: 3$ \\
$620(\mathrm{a})$ & $18: 1 / 18: 1$ \\
$620(\mathrm{~b})$ & $18: 0 / 18: 1$ \\
622 & $18: 1 / 18: 0$ \\
\hline
\end{tabular}

Identified FAs: $16: 0$ palmitic, 16:1 palmitoleic, 17:0 margaric, 17:1 margaroleic, 18:0 stearic, 18:1 oleic, 18:2 linoleic, 18:3 linolenic.

of several insect species (Beenakkers et al., 1985; Arrese \& Wells, 1997) most of the DGs contain acyls in the $s n-1,2-\mathrm{DG}$ position.

The percentage of individual DGs present in the haemolymph of brachypterous and macropterous bugs after injection with either 10 pmol Pyrap-AKH or PeramCAH-II is shown in Fig. 3. The results were calculated from the ESI-MS ${ }^{2}$ analyses, in which the subtraction of the total DG level in resting (time 0) and treated (90 min later) bugs were considered to be $100 \%$ (e.g. $100 \%$ was total elevation of all DGs after the hormonal treatment). Injection of the solvent (control treatment) had no effect (data not shown for clarity). The results revealed that just four or five DGs $(592,616,618,620$ and less so $594 \mathrm{Da})$ were responsible for about $90 \%$ of the increase in lipids induced by the injection of hormones in both groups of bugs studied; these DGs contained $\mathrm{C} 18$ and $\mathrm{C} 16$ FAs only. Fig. 3A also shows there were no statistical difference in the DGs mobilized by Pyrap-AKH and Peram$\mathrm{CAH}-\mathrm{II}$ in brachypterous bugs, whereas in macropterous bugs (Fig. 3B) Pyrap-AKH significantly mobilized more 616 Da DG and Peram-CAH-II more of the 594 and 620 Da DGs.

\section{FA composition of TGs in the FBs of brachypterous and macropterous bugs}

The theoretical FA composition of TGs from FBs of brachypterous and macropterous bugs is presented in Table 2 (raw HPLC/ESI-MS data are shown just for brachypterous FB TGs in Fig. 5B - see below). These results indicate that most TGs are present in low amounts and only 3 TGs of mws 878,880 and 882 Da (the last two exist in two molecular forms), each make up more than $10 \%$ of the total the TG content of FB (Table 2). It is 
TABLE 2. Characterization of the TGs in the FBs of brachypterous and macropterous bugs. For clarity only the TGs with a relative percentage higher than 0.5 were included in the analysis. The TGs whose two different theoretical FA variants within the TG molecules could not be distinguished are marked by *; their amount is expressed as a percentage deduced from the ESI-MS ${ }^{2}$ fragmentation.

\begin{tabular}{|c|c|c|c|c|c|}
\hline \multirow{2}{*}{ TG mw (Da) } & \multirow{2}{*}{\multicolumn{3}{|c|}{$\begin{array}{l}\text { Theoretical FA composition and percentage } \\
\text { of different FA variants* }\end{array}$}} & \multicolumn{2}{|c|}{ Relative $\% \pm$ SD of TG } \\
\hline & & & & Brachypterous bugs $(n=12)$ & Macropterous bugs $(\mathrm{n}=12)$ \\
\hline 810 & $13: 1 / 18: 2 / 18: 2$ & & & $0.62 \pm 0.33$ & $2.07 \pm 0.85$ \\
\hline 812 & $13: 1 / 18: 1 / 18: 2$ & & & $0.48 \pm 0.26$ & $1.44 \pm 0.64$ \\
\hline 830 & $16: 0 / 16: 0 / 18: 2$ & & & $2.52 \pm 0.99$ & $2.22 \pm 0.76$ \\
\hline 832 & $16: 0 / 16: 0 / 18: 1$ & & & $0.64 \pm 0.28$ & $0.68 \pm 0.25$ \\
\hline 854 (a) & $16: 1 / 18: 1 / 18: 2$ & & & $4.93 \pm 2.90$ & $5.58 \pm 2.24$ \\
\hline 854 (b) & $16: 0 / 18: 2 / 18: 2$ & & & $1.50 \pm 1.75$ & $1.49 \pm 0.58$ \\
\hline 856 & $16: 0 / 18: 1 / 18: 2$ & & & $7.58 \pm 2.51$ & $6.94 \pm 2.16$ \\
\hline 858 (a) & $16: 0 / 18: 1 / 18: 1$ & & & $1.84 \pm 1.03$ & $1.36 \pm 0.45$ \\
\hline 858 (b) & $16: 0 / 18: 0 / 18: 2$ & & & $2.60 \pm 1.28$ & $1.91 \pm 0.65$ \\
\hline 860 & $16: 0 / 18: 0 / 18: 1$ & & & $0.94 \pm 0.73$ & $0.46 \pm 0.18$ \\
\hline 864 & $17: 2 / 18: 2 / 18: 2$ & & & $0.69 \pm 0.37$ & $0.48 \pm 0.22$ \\
\hline 866 & $17: 1 / 18: 2 / 18: 2$ & $17: 2 / 18: 1 / 18: 2$ & $66: 33^{*}$ & $1.34 \pm 0.50$ & $1.18 \pm 0.41$ \\
\hline 868 (a) & $17: 1 / 18: 1 / 18: 2$ & & & $0.68 \pm 0.35$ & $0.59 \pm 0.20$ \\
\hline 868 (b) & $16: 0 / 18: 2 / 19: 2$ & & & $1.37 \pm 0.65$ & $1.18 \pm 0.42$ \\
\hline 870 & $17: 1 / 18: 0 / 18: 2$ & $16: 0 / 18: 1 / 19: 2$ & $50: 50^{*}$ & $0.71 \pm 0.41$ & $0.50 \pm 0.18$ \\
\hline 876 & $18: 1 / 18: 2 / 18: 4$ & & & $0.75 \pm 0.36$ & $0.54 \pm 0.26$ \\
\hline 878 & $18: 2 / 18: 2 / 18: 2$ & $18: 1 / 18: 2 / 18: 3$ & $50: 50^{*}$ & $12.16 \pm 4.74$ & $17.44 \pm 5.44$ \\
\hline 880 (a) & $18: 1 / 18: 2 / 18: 2$ & & & $13.17 \pm 4.68$ & $11.67 \pm 3.58$ \\
\hline 880 (b) & $18: 2 / 18: 2 / 18: 1$ & & & $2.34 \pm 1.29$ & $5.08 \pm 1.65$ \\
\hline 882 (a) & $18: 1 / 18: 1 / 18: 2$ & & & $6.28 \pm 2.57$ & $5.95 \pm 1.84$ \\
\hline 882 (b) & $18: 0 / 18: 2 / 18: 2$ & & & $5.75 \pm 3.17$ & $4.59 \pm 1.41$ \\
\hline 884 (a) & 18:1/18:1/18:1 & & & $1.25 \pm 1.51$ & $0.92 \pm 0.74$ \\
\hline 884 (b) & $18: 0 / 18: 1 / 18: 2$ & & & $4.91 \pm 2.32$ & $3.23 \pm 1.07$ \\
\hline 886 (a) & 18:0/18:1/18:1 & & & $0.98 \pm 0.60$ & $0.54 \pm 0.19$ \\
\hline 886 (b) & 18:0/18:0/18:2 & & & $1.74 \pm 1.05$ & $0.55 \pm 0.22$ \\
\hline 892 & $18: 2 / 18: 2 / 19: 2$ & & & $2.28 \pm 0.94$ & $2.53 \pm 0.79$ \\
\hline 894 (a) & $18: 2 / 18: 2 / 19: 1$ & & & $2.07 \pm 1.05$ & $2.47 \pm 0.80$ \\
\hline 894 (b) & $18: 1 / 18: 2 / 19: 2$ & & & $1.33 \pm 1.52$ & $1.17 \pm 1.21$ \\
\hline 896 & $18: 1 / 18: 2 / 19: 1$ & & & $1.83 \pm 0.95$ & $1.29 \pm 0.40$ \\
\hline
\end{tabular}

Identified FAs: 13:1 tridecenoic, 16:0 palmitic, 16:1 palmitoleic, 17:0 margaric, 17:1 margaroleic, 17:2 heptadecadienoic, 18:0 stearic, 18:1 oleic, 18:2 linoleic, 18:3 linolenic, 19:1 nanodecenoic, 19:2 nanodecadienoic.

interesting to note that all of these three TGs contained just C18 (mostly) unsaturated FAs. There are no FB DGs in Table 2 because they make up only $2 \%$ of the FB lipids and were omitted for reasons of clarity. The relative percentages of FAs in the FB TGs of both groups of bugs tested were derived from the data in Table 2 and compared with similarly calculated (for details see Material and Methods) percentage FA content of hormonally mobilized DGs. The results presented in Fig. 4 (the 17:2 and 18:4 FAs were omitted because they are present in negligible amounts - see Table 2) indicate that (1) as mentioned above the $\mathrm{C} 16$ and $\mathrm{C} 18$ FAs have a crucial role - FAs with a carbon chain longer than 18 and shorter than 16 do not have a role in lipid mobilization, although present in FBs; (2) there are significant differences in the relative percentage of FA in the TG pool in the $\mathrm{FB}$, and DG pool in the haemolymph of hormonally treated bugs of both groups: in other words percentage composition of the mobilized FAs in the haemolymph does not reflect that in the FB, which indicates that only particular FAs are mobilized; (3) this preferential mobilization is similar in both brachypterous and macropterous bugs for which only quantitative differences were recorded; (4) the differences in FA levels in hormonally mobilized DGs and those in the FB TGs were recorded for palmitic (16:0), stearic (18:0), oleic (18:1), linoleic (18:2) and linolenic (18:3) acids, whereas the levels of palmitic and linoleic acids were higher, and those of stearic, oleic and linolenic acids were lower in the haemolymph than in the FB of hormonally treated bugs.

Statistical evaluation of the effect of individual hormones on the mobilization of particular FAs (Table 3) indicates there was no significant difference in the effect of Pyrap-AKH and Peram-CAH-II in brachypterous bugs, but in macropterous bugs Pyrap-AKH mobilized more linoleic acid and Peram-CAH-II more oleic acid (see Table 3 and Fig. 4). These results do not contradict the results presented in Fig. 3 for the relationships for the DG level.

Fig. 4 reveals that linoleic acid was the most abundant FA involved in the AKH induced mobilization of DGs, making up $60 \%$ of the lipids mobilized by Pyrap-AKH 

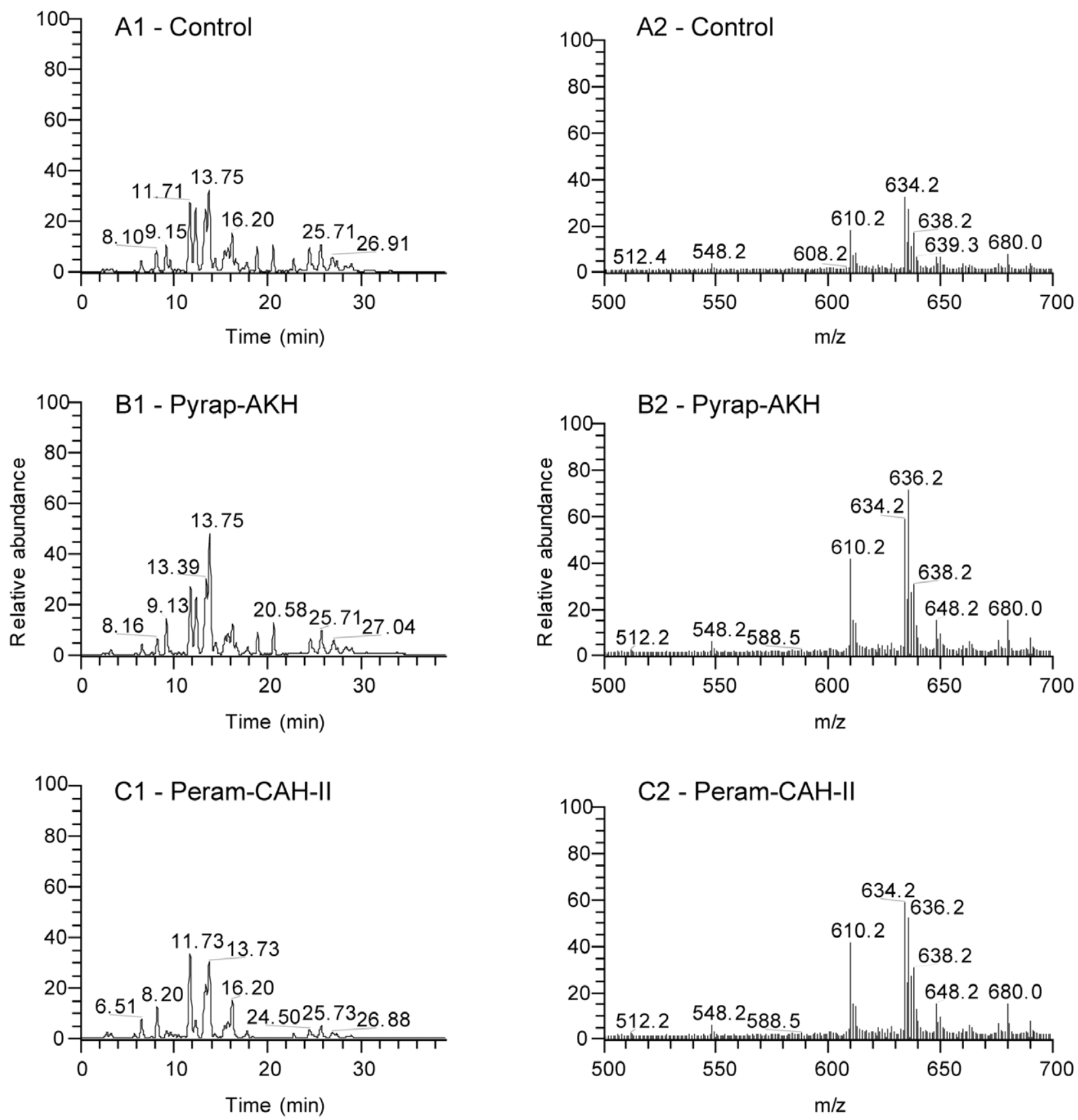

Fig. 1. The HPLC/ESI-MS elution profiles of DGs in lipid extracts of haemolymph $(10 \mu \mathrm{l})$ obtained from reproductive brachypterous $P$. apterus $(\mathrm{A} 1-\mathrm{C} 1)$ and their corresponding ESI-TIC spectra (A2-C2) showing molecular species composition of putative DGs. A retention time window of 7.5-21.5 min, corresponding to the elution of the DG standards, was used for the MS analysis. The DGs were ionized as ammonium adducts $\left[\mathrm{M}+\mathrm{NH}_{4}\right]^{+}([$molecular mass $+18 \mathrm{Da}])$ and their identity deduced from the CID MS ${ }^{2}$ spectral fragmentations (data not shown). Panels A1 + A2 are the DGs in the haemolymph of bugs treated with Ringer solution, panels B1 + $\mathrm{B} 2$ those in the haemolymph after treatment with Pyrap-AKH and $\mathrm{C} 1+\mathrm{C} 2$ after treatment with Peram-CAH-II.

and $50 \%$ of that by Peram-CAH-II. Nevertheless, the linoleic acid content of the FB is around $45 \%$, which indicates the importance of this FA in energy metabolism and the specificity of the AKHs, especially Pyrap-AKH, in its mobilization.

\section{A comparison of the lipids in linden seeds and the FB}

The lipids in linden seeds were only compared with those in the FBs of brachypterous bugs because 10-day old adult macropterous bugs do not feed. The HPLC/ESI-MS elution profiles (Fig. 5) showed a clear dominance of TGs in linden seeds and a negligible presence of DGs (RT = 7.5-21.5 min, Fig. 5A). The quantified ESI-TIC spectra of the TGs (Fig. 5C and 5D) are illustrated in Fig. 6 and as in the previous analyses TGs with a low percentage incidence $(<1 \%)$ were omitted. The TGs with mws of 878,880 and 882 Da were the most abundant in linden seeds and the FB. However, there are statistically significant differences in the quantity of particular TGs in linden seeds and FBs, with predominance the TGs of mws $880,886,892$ and $894 \mathrm{Da}$ in the FBs and 

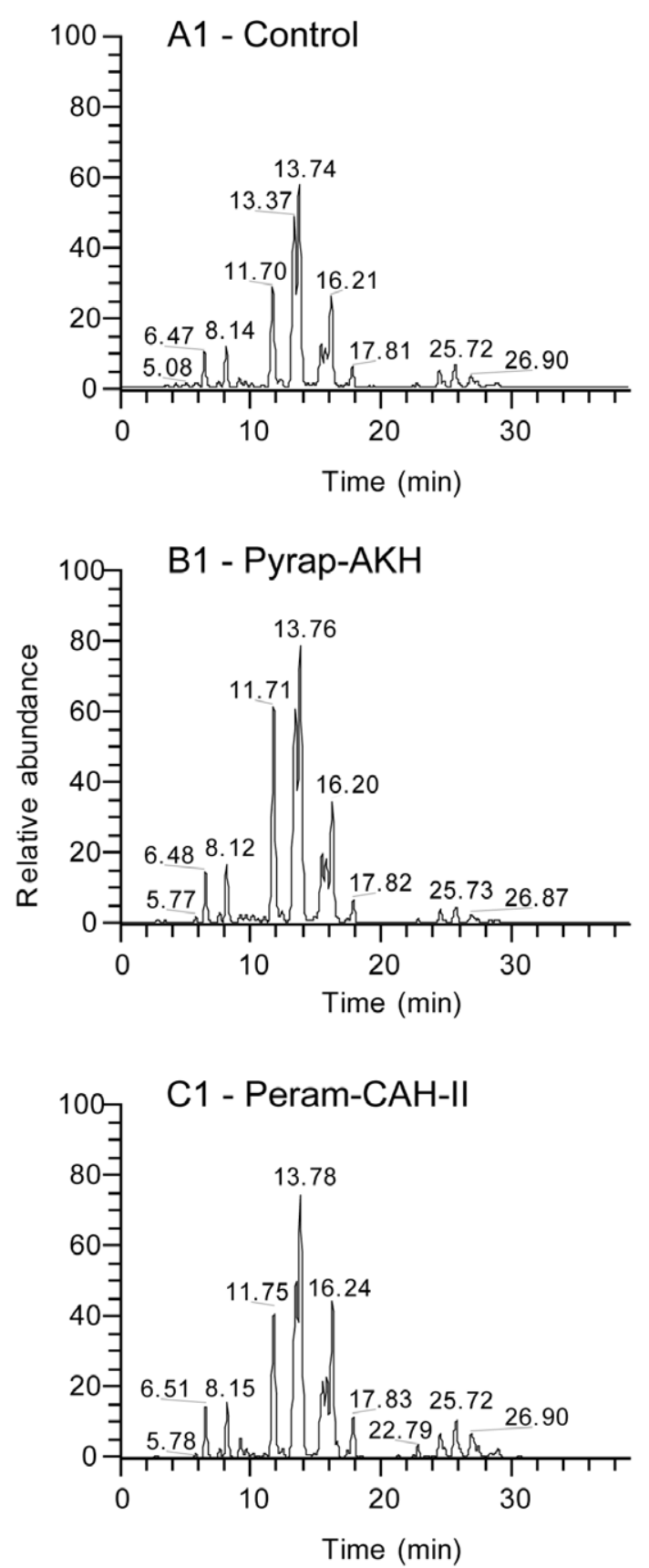
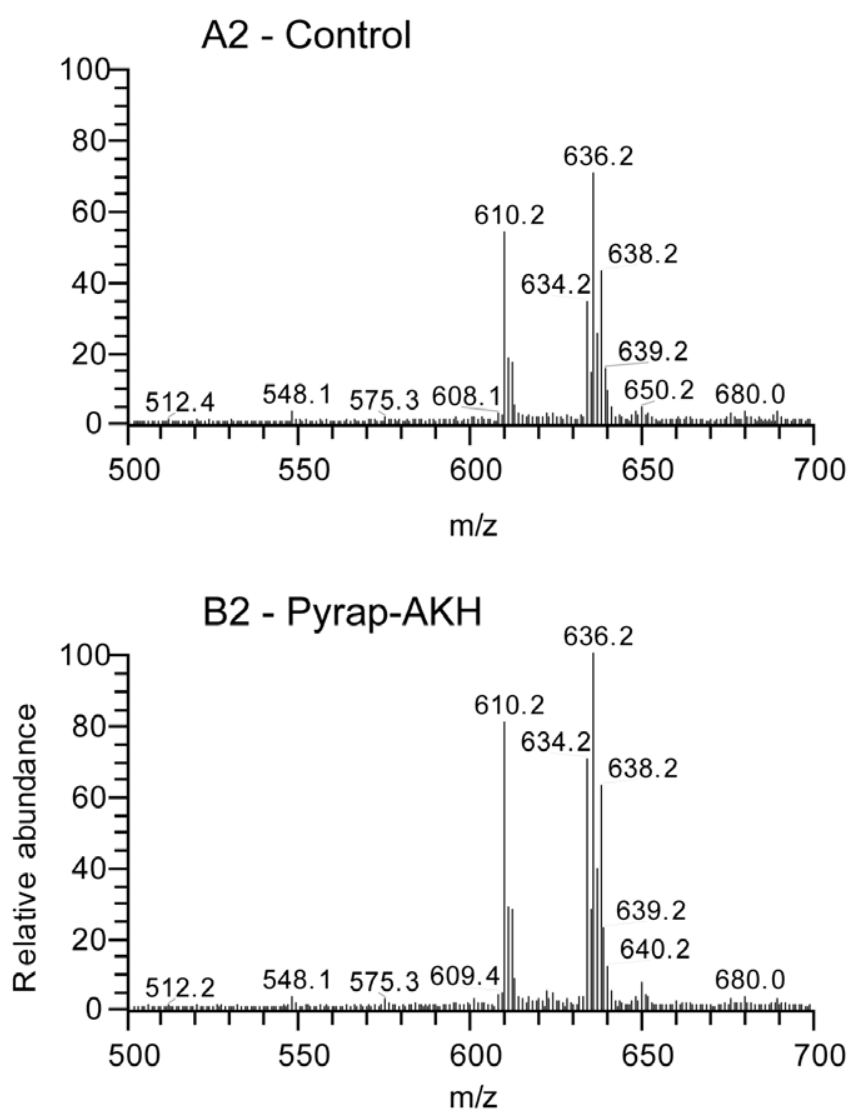

\section{C2 - Peram-CAH-II}

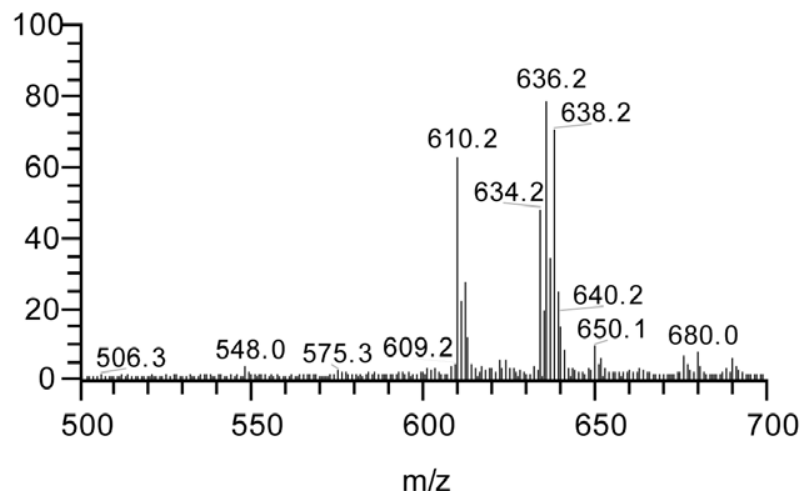

Fig. 2. The HPLC/ESI-MS elution profiles of DGs in lipid extracts of haemolymph (10 $\mu 1)$ from macropterous $P$. apterus (A1-C1) and their corresponding ESI-TIC spectra (A2-C2) showing molecular species composition of putative DGs. For details see Fig. 1.

those with mws $(826,852) 864,866,868,876,908$ and $910 \mathrm{Da}$ in linden seeds (Fig. 6A). A calculation of relative percentage of FAs in linden seed and FB TGs (see above and also Materials and Methods) revealed a remarkable accumulation of palmitic (16:0), stearic (18:0), oleic (18:1) and linolenic (18:3) acids in the FB (Fig. 6B). The level of the most abundant FA, linoleic acid (18:2), was highest in linden seed and FB TGs. The level of palmitoleic (16:1), margaroleic (17:1) and norlinoleic (17:2) acids was significantly lower in FB TGs. These results accord with the spectra of AKH mobilized FAs illustrated in Fig. 4.

\section{DISCUSSION}

Differences in the AKH-mobilized lipids in P. apterus and other insects

It is well documented in a series of papers that the firebug $P$. apterus obtains most of the energy it needs from lipids (Martin, 1969a, b; Socha \& Kodrík, 1999) and that firebugs injected with of Pyrap-AKH and PeramCAH-II mobilize mostly lipids (Kodrík et al., 2000, 2002, 2005; Socha et al., 2005, etc.) while there is no or only a negligible mobilization of glycides (Socha et al., 2004). Therefore, this bug appears to be a good model for a detailed study of the spectrum of lipids mobilized by AKH. 

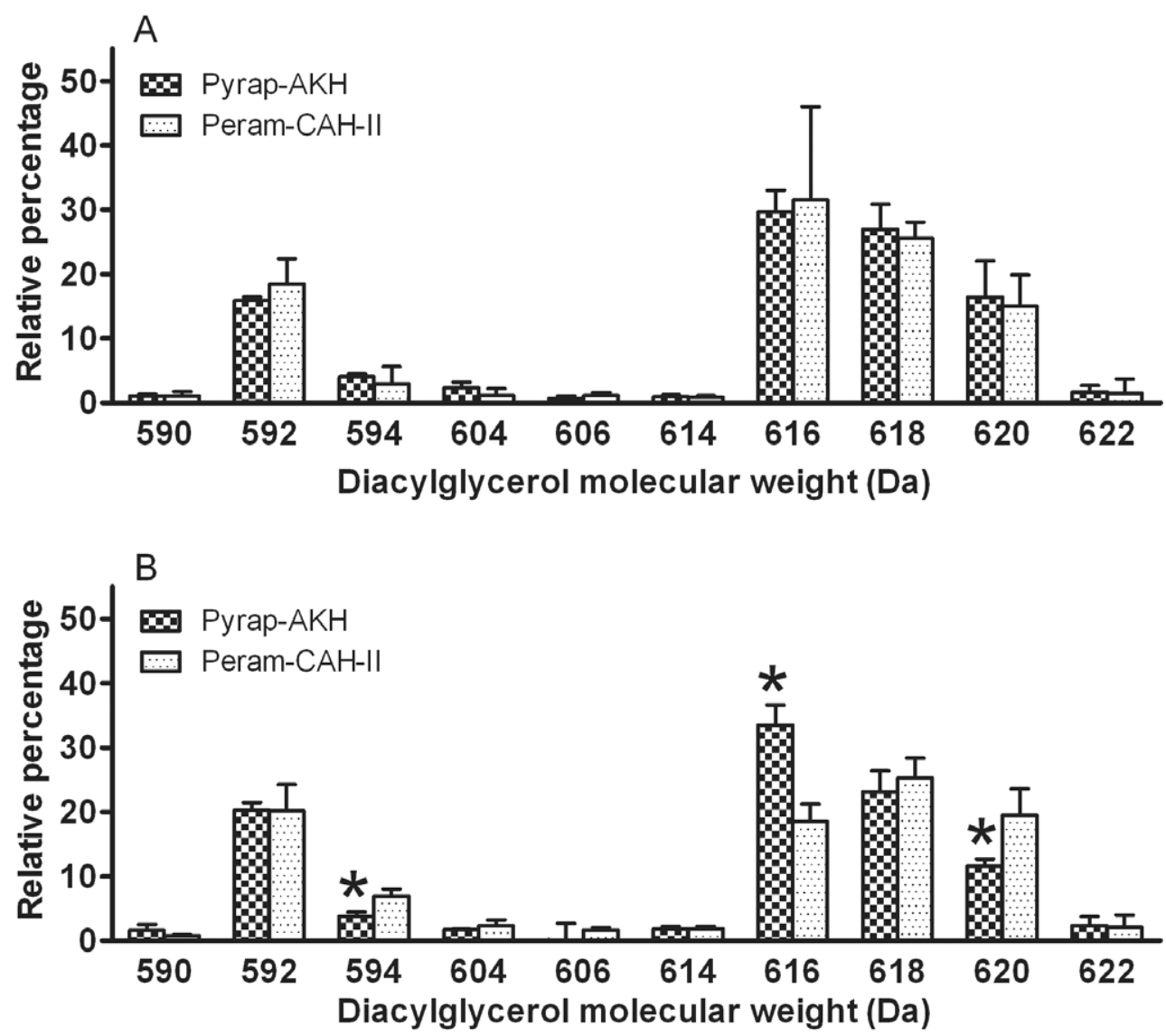

Fig. 3. Relative percentage of the individual DG species participating in total elevation of haemolymph lipids $(=100 \%)$ of brachypterous (A) and macropterous (B) bugs injected with Pyrap-AKH and Peram-CAH-II. Theoretical FA composition of the DG species is summarized in Table 1. The effect of injecting only solvent was negligible (data not shown), see text. Statistically significant differences at the 5\% level (DG mobilized by Pyrap-AKH vs. DG mobilized by Peram-CAH-II) evaluated using t-tests are indicated by *; the bars represent $\mathrm{SD}, \mathrm{n}=3$.

TABLE 3. Test for FA preference: a statistical evaluation of relative percentage of the FAs (see Fig. 4) present in the DGs detected in haemolymph after the separate Pyrap-AKH and Peram-CAH-II treatments. The statistics compares the difference in effect of individual hormones on the mobilization of particular FAs. S - significant difference; NS - no significant difference; 13:1, 19:0 and 19:1 FAs were not detected in haemolymph DGs.

\begin{tabular}{ccc}
\hline \multirow{2}{*}{ FA structure } & \multicolumn{2}{c}{$\begin{array}{c}\text { Student t-test - statistical significance at 5\% } \\
\text { level: Pyrap-AKH vs. Peram-CAH-II }\end{array}$} \\
\cline { 2 - 3 } & Brachypterous bugs & Macropterous bugs \\
\hline $13: 1$ & - & - \\
$16: 0$ & NS & NS \\
$16: 1$ & NS & NS \\
$17: 0$ & NS & NS \\
$17: 1$ & NS & NS \\
$18: 0$ & NS & NS \\
$18: 1$ & NS & S \\
$18: 2$ & NS & S \\
$18: 3$ & NS & NS \\
$19: 1$ & - & - \\
$19: 2$ & - & - \\
\hline
\end{tabular}

Identified FAs: 13:1 tridecenoic, 16:0 palmitic, 16:1 palmitoleic, 17:0 margaric, 17:1 margaroleic, 18:0 stearic, 18:1 oleic, 18:2 linoleic, 18:3 linolenic, 19:0 nanodecanoic, 19:1 nanodecenoic.
This study revealed that DGs are selectively mobilized by AKH. The results demonstrated that mainly four DGs (592, 616, 618 and $620 \mathrm{Da})$ were responsible for the majority of DG mobilization. These DGs contain only C16 and C18 FAs, which is not surprising, as eukaryotic FA-synthases mainly synthesize C16 saturated FAs with smaller amounts of $\mathrm{C} 14$ and C18 FAs. The specificities of the chain-elongating and chain-terminating enzymes complement each other perfectly, ensuring that these FAs are the major products (Rangan \& Smith, 2002). This does not account for the differences in the relative levels of particular FAs in TGs in FB and DGs in the haemolymph, which partly indicates that particular AKHs mobilize particular FAs. The results indicate that the levels of palmitic (16:0) and linoleic (18:2) FAs were higher, and stearic (18:0), oleic (18:1) and linolenic (18:3) FAs lower in haemolymph of both morphs treated with both Pyrap-AKH and Peram-CAH-II, than in the FB. The reasons for this selectivity are not clear, however, a similar AKH-induced mobilization of particular FAs was recently recorded in L. migratoria (Tomčala et al., 2010). All three Locmi-AKHs selectively mobilized DGs that contain oleic (18:1) and linolenic (18:3) acids; moreover AKH-I selectively mobilized linoleic acid (18:2) containing DGs, and AKH-II and AKH-III stearic acid (18:0) 

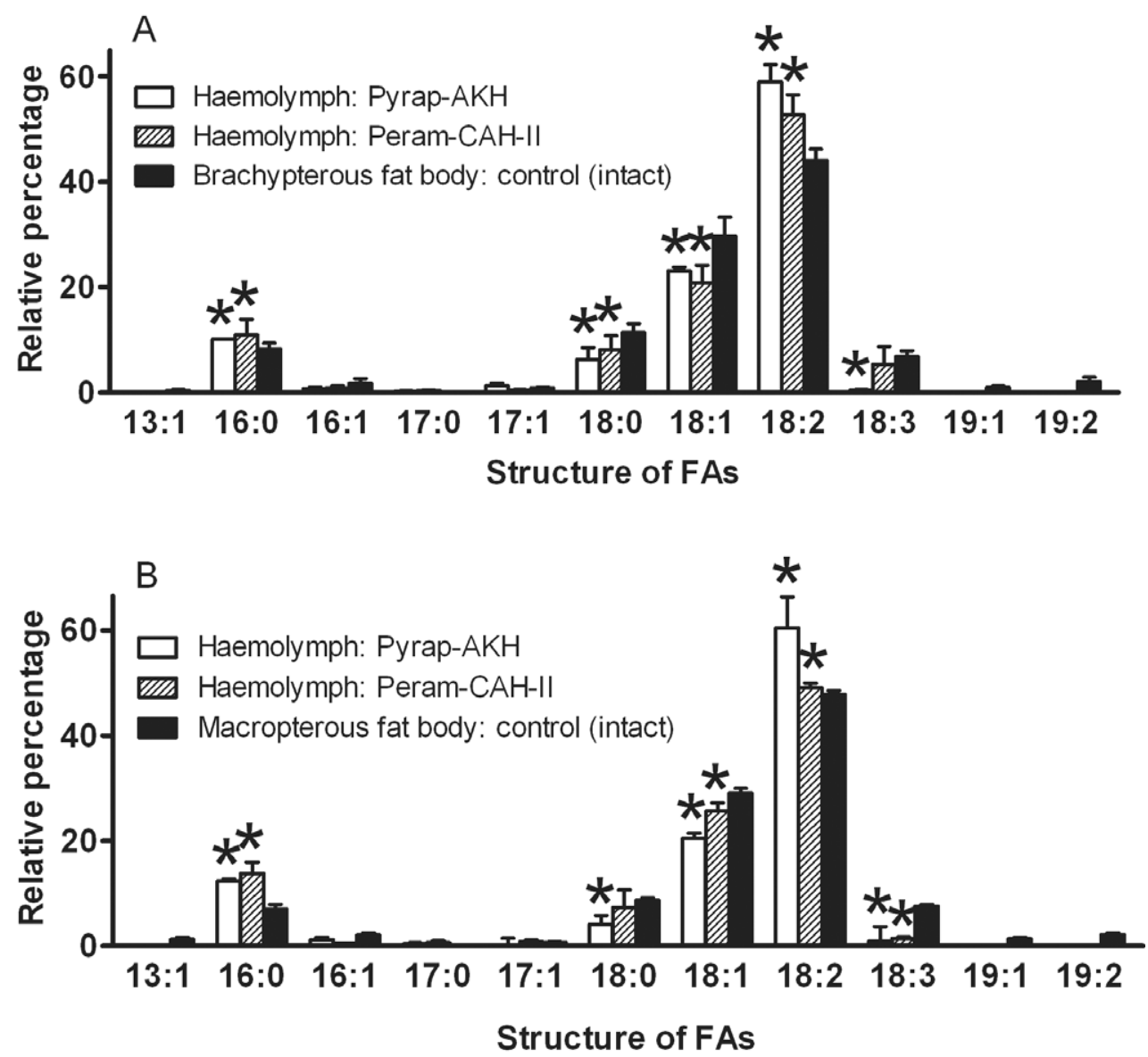

Fig. 4. Relative percentage of the FAs participating in total elevation of haemolymph DGs $(=100 \%)$ of brachypterous (A) and macropterous (B) bugs injected with Pyrap-AKH and Peram-CAH-II, and their comparison with FAs in the corresponding FB TGs. Statistically significant differences at the $5 \%$ level (FAs in haemolymph DGs vs. FAs in FB TGs) evaluated using t-tests are indicated by *. The TG FAs make up $100 \%$ of the total FAs (including those present in negligible amounts and not shown in Table 2). The bars represent $\mathrm{SD}, \mathrm{n}=3$ for haemolymph samples and $\mathrm{n}=12$ for FB samples.

containing DGs. Relative percentage of palmitic acid $(16: 0)$ in the haemolymph of Locmi-AKH treated insects was lower than in the FB. It is evident that in both species (i.e. P. apterus and L. migratoria) different FAs are mobilized, but whether it depends on differences in their biology, food or living conditions, or some biochemical reason such as a difference in the composition of the AKHs, is unknown.

The FA composition of acylglycerols appears to be different in the FB and haemolymph of several species of insect (Beenakkers et al., 1985). This disparity is explained by the putative existence of two different acylglycerol pools in the FB and their complicated incorporation into DGs. Notwithstanding, the difference in the FA composition of released and stored glycerides indicates there is a selective release of glycerides with particular FA compositions (Keeley, 1985). Alternatively it could indicate the release of $s n-1,2-\mathrm{DG}$ from FB TGs by the specific hydrolysis of the FA at the $s n-3$ position of the TGs (Van der Horst, 1982) and that the FA composition of the DG could be a result of the non-random distribution of FAs over the three stereospecific positions in the TG. Moreover, the absence of FAs with $\mathrm{C}>18$ in the DGs released into the haemolymph recorded by several authors (this study; Martin, 1969a; Beenakkers \& Scheres, 1971; Beenakkers et al., 1985; Tomčala et al., 2010) may be due to their position at $s n-3$.

Both $P$. apterus AKHs similarly mobilized particular FAs (haemolymph DG FAs vs. FB TG FAs - as discussed in the second paragraph), but in the macropterous morph there were significant quantitative differences between the hormones. Intensity of mobilization of linoleic acid (18:2) was significantly higher following injection with Pyrap-AKH than Peram-CAH-II, while intensity of oleic acid (18:1) mobilization by Peram-CAH-II was higher than by Pyrap-AKH in this morph. At present it is not possible to offer a satisfactory explanation of this phenomenon. However, a preference of individual AKHs for particular FA species is more obvious in $L$. migratoria. It seems there is at least a partial specificity of individual locust AKHs for certain FAs, with LocmiAKH-I preferentially mobilizing unsaturated FAs (mostly linoleic acid) and AKH-II and AKH-III saturated FAs (mostly stearic) (Tomčala et al., 2010). This accords with the Murata \& Tojo (2002) finding that in locusts unsaturated FAs in TGs are mainly used as an energy source during flight, and that Locmi-AKH-I is more involved in lipid mobilization during flight than Locmi-AKH-II and 

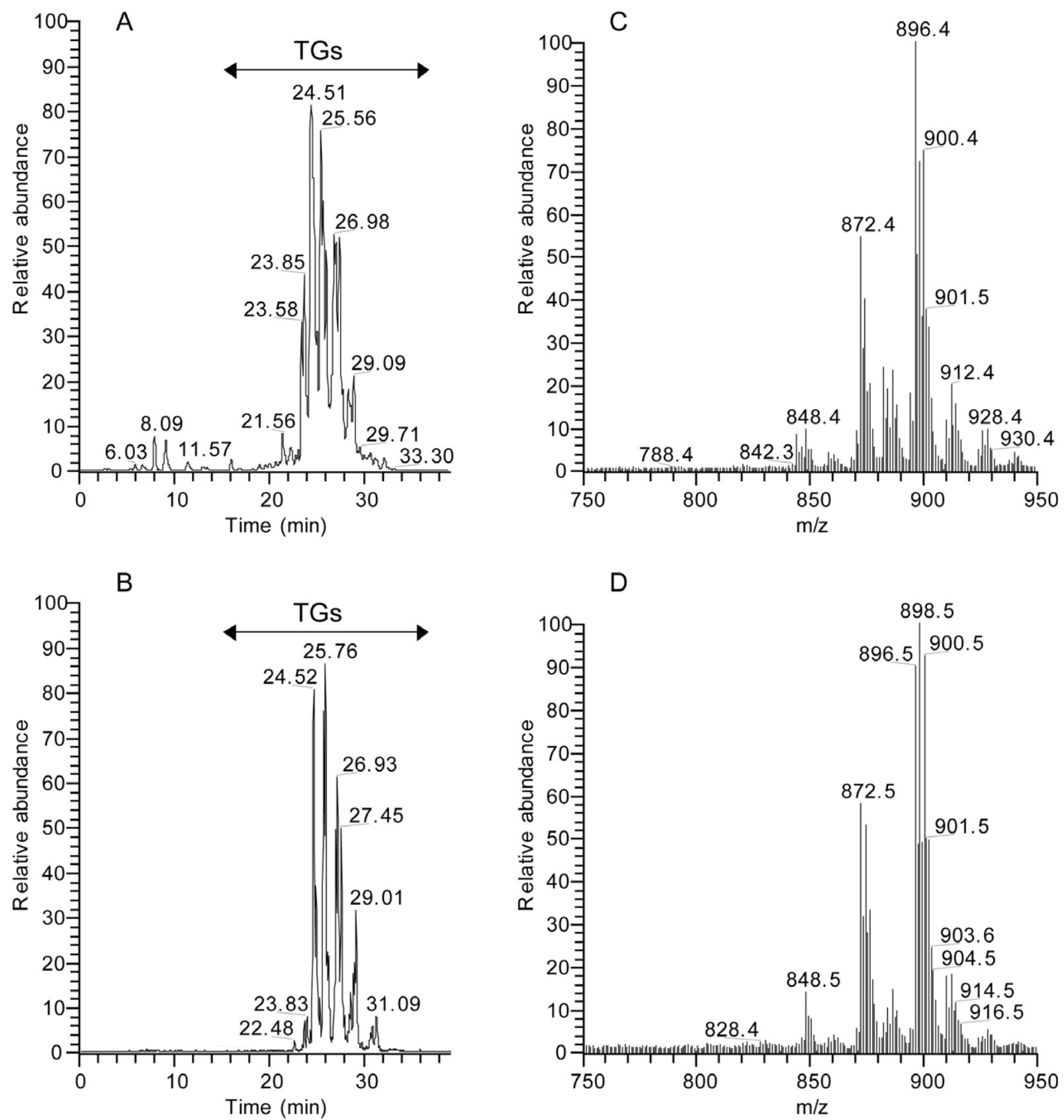

Fig. 5. The HPLC/ESI-MS elution profiles of TGs in lipid extracts of linden seeds (A) and FB of brachypterous P. apterus (B; see also Table 2, penultimate column) and their corresponding ESI-TIC spectra (C, D) showing molecular species of TGs present in linden seed and FB, respectively. The TGs were ionized as ammonium adducts $\left[\mathrm{M}+\mathrm{NH}_{4}\right]^{+}([$molecular mass $+18 \mathrm{Da}])$ and their identity deduced from the CID MS² fragments by means of Trigly $\mathrm{APCI}^{\circ}$ software (data not shown).

-III (Goldsworthy, 1994). The situation in P. apterus is more complicated due to the fact that the phenomenon was recorded only for the macropterous morph and no DG or FA preferences were found for the brachypterous morph. These wing morphs differ in various physiological and behavioural parameters, e.g. the length of preoviposition period (Socha \& Šla, 1996), feeding activities, energy reserves in the FB (Socha et al., 1997, 1998) and adipokinetic response (Socha \& Kodrík, 1999). Mobilization of lipids by AKH in flightless macropters (Maxová et al., 2001) is positively correlated with walking activity, and also the AKH content of the CNS
(Kodrík et al., 2003). One can speculate that the greater level of AKH mobilization of DGs or FAs in the macropterous morph reflect its higher general metabolic, dispersal and walking activities.

The most abundant FA in P. apterus, both among the AKH mobilized FAs and those stored in the FB, is linoleic acid (18:2). Percentage of linoleic acid varied around $45 \%$ in the FB and made up $50-60 \%$ of the lipids mobilized. The importance of linoleic acid in P. apterus was recorded a long time ago by Martin (1969a) who finds that it made up 55-60\% of the lipids in the FB of adults during the first 5 days of their life and fluctuated around 


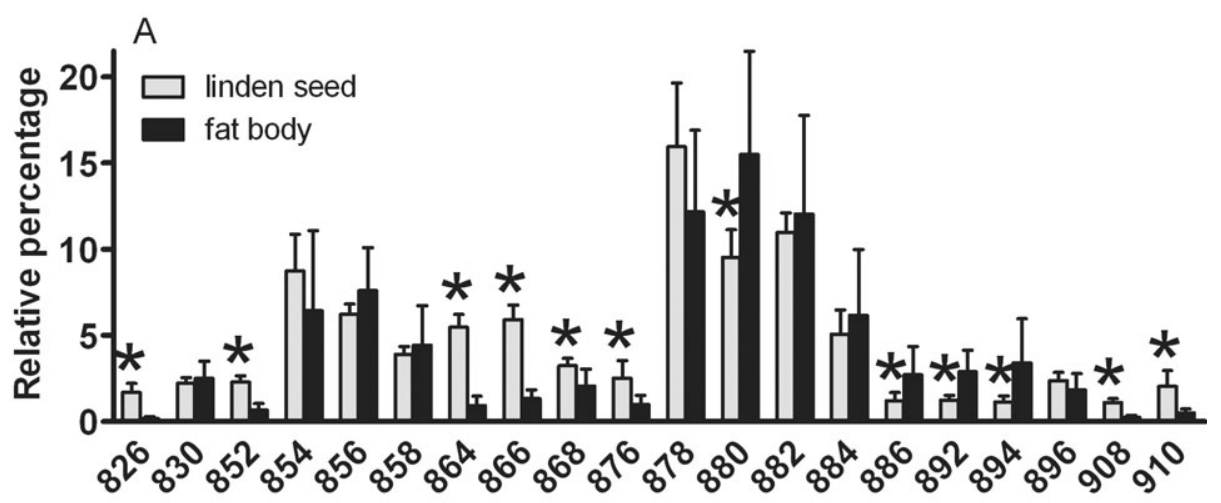

Triacylglycerol molecular weight (Da)

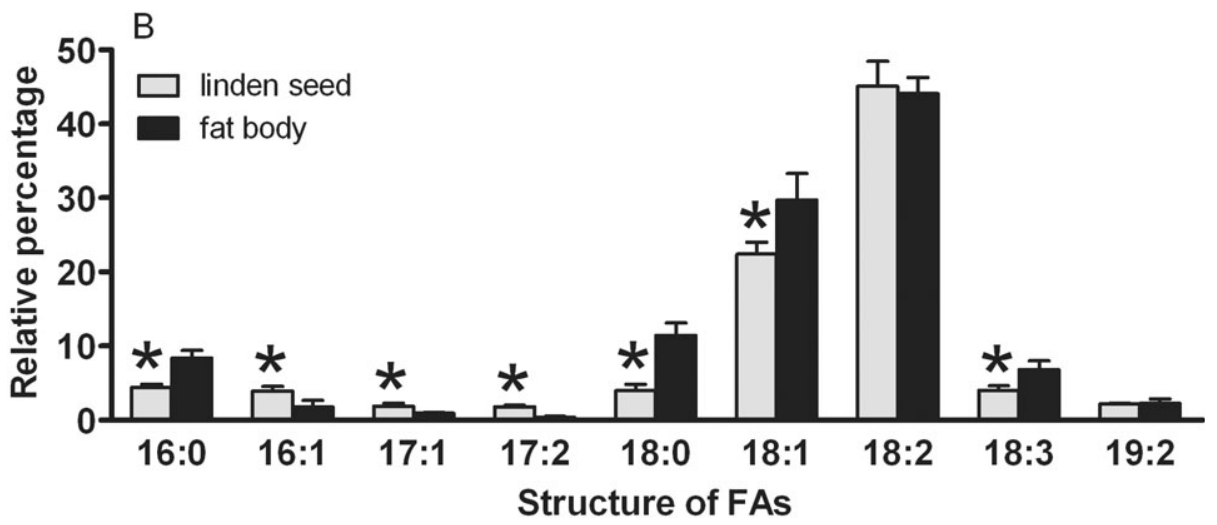

Fig. 6. Relative percentage of the TGs (A) and FAs (B) present in lipid extracts from linden seeds and FBs of brachypterous $P$. apterus FBs. Statistically significant differences at the 5\% level (TGs or FAs in linden seeds vs. TGs or FAs in brachypterous FBs) evaluated using t-tests are indicated by *. The bars represent $\mathrm{SD}, \mathrm{n}=7$ for linden seed samples and $\mathrm{n}=12$ for FB samples.

$50-55 \%$ in the ovaries in the $3^{\text {rd }}-5^{\text {th }}$ day of adult life. Those results suggest that linoleic acid might play a significant role in $P$. apterus in the most energetic demanding processes in insects controlled by AKH: locomotion and vitellogenin synthesis. In other insects oleic acid (18:1) has this role. It is responsible for $35-40 \%$ of the AKH induced lipid mobilization in L. migratoria, and the content of this $\mathrm{FA}$ in the $\mathrm{FB}$ is about $25 \%$, which is identical to that of another frequent FA, palmitic acid (16:0), in this species (Beenakkers \& Scheres, 1971; Tomčala et al., 2010). Nevertheless, oleic acid is the second most abundant FA in $P$. apterus (this paper) reaching about $20-25 \%$ in mobilized DGs and about $30 \%$ in FB TGs. The percentage of oleic acid in the FB lipids is generally higher in potential migrants than in solitary individuals, as in the gregarious and solitary phases of Schistocerca gregaria. The DGs that contain oleic acid and unsaturated $\mathrm{C} 18$ linoleic and linolenic acids, increase significantly in the haemolymph during flight in this species. They fluctuated significantly not only in relation to phase, but also to age (Schneider \& Dorn, 1994). A high content of the oleic FA is recorded in the FBs of several other insects including the beetle Zophobas atratus (Howard \& Stanley-Samuelson, 1996). Oleic acid is also the most common DG-lipophorin FA in the bloodsucking bug Panstrongylus megistus (Canavoso et al., 2004).

\section{Lipids in $P$. apterus and its food}

The digestion and absorption of lipids is analogous in insects and vertebrates, but with some important differences. Insects do not have bile salts, but have developed alternative strategies: the strategies include the use of lumenal glycolipids and the formation of fatty acyl-amino acid complexes, and FA and lysophospholipid micelles, which facilitate lipid solubility (Turunen \& Crailsheim, 1996). Their mid gut cells produce lipases that hydrolyze the dietary TGs forming monoacylglycerols and free FAs (Hoffman \& Downer, 1979). These FAs are absorbed and converted into DGs in the mid gut cells using the phosphatidic acid pathway (Canavoso \& Wells, 2000). Then the DGs are rapidly converted into TGs, which serve as a reservoir for absorbed FAs; alternatively the DGs are transported directly to the FB via haemolymph (Canavoso $\&$ Wells, 2000) where they are also converted into TGs. Their composition largely depends on their diet and can to some degree be changed experimentally. Beenakkers \& Scheres (1971) fed L. migratoria various diets containing different lipids, which revealed that the main differences between the diet and FB lipids is restricted to unsaturated C18 FAs. There is a fair correlation between the percentage of oleic (18:1) and linoleic (18:2) FAs in the diet and FB, while the amount of linoleic acid in the FB is not influenced by differences in diet. 
The laboratory culture of $P$. apterus used in this study was strictly monophagous as they were fed exclusively on linden seeds. Lipid analysis of the seeds done using thin layer chromatograph with silica gel-G (Martin, 1969a) and HPLC/ESI-MS in the present study, revealed an almost identical composition of the main lipid components. Comparison of the lipid spectra for the seeds and the FB revealed they have similar TG and FA profiles, but with significant differences (see Fig. 6). In both the TGs 878-884 Da (containing only C18 mostly unsaturated FAs) and TGs 854 and $856 \mathrm{Da}$ (containing both saturated and unsaturated $\mathrm{C} 16$ and $\mathrm{C} 18$ ) were the most abundant. The results also revealed that the TGs with $\mathrm{C} 18$ FAs (mostly unsaturated) were preferred, while the content of TGs containing abnormal C17 FAs (864, 866 and $868 \mathrm{Da}$ ) was significantly lower in the FB than in linden seeds. It is evident that $P$. apterus FB accumulates FAs that have an important role in lipid mobilization: palmitic (16:0), stearic (18:0), oleic (18:1) and linoleic (18:3) FAs, and that the main P. apterus FA - linoleic acid (18:2) - is abundant in both its diet and FB. Its abundance in linden seeds might be one of the reasons why linoleic acid is important in $P$. apterus lipid metabolism.

In summary, an analysis of the lipids mobilized by AKHs in P. apterus FB and transported in the haemolymph revealed that $\mathrm{C} 16$, to a small extent, and mainly (unsaturated) C18 FAs are the dominant shuttles involved in the transport of lipids. In addition, to a certain extent only particular FAs are mobilized by the $P$. apterus AKHs, with the preference almost identical but for a few quantitative differences in both macropterouss and brachypterous bugs. Separate action of the Pyrap-AKH and the Peram-CAH-II only revealed distinct changes in FA profiles in macropterous bugs. It seems that the FAs participating in AKH-induced lipid mobilization are preferentially absorbed from linden seeds and accumulated in the FB.

ACKNOWLEDGEMENTS. This study was supported by the grants Nos P501/10/1215 (DK) and P502/10/1734 from the Czech Science Foundation, and by project No. Z50070508 of the Institute of Entomology, funded by the Academy of Sciences of the Czech Republic. The authors thank D. Rienesslová for her technical assistance and P. Jedlička (Institute of Entomology) for critically reading of the MS.

\section{REFERENCES}

Arrese E.L. \& Soulages J.L. 2010: Insect fat body: energy, metabolism, and regulation. Annu. Rev. Entomol. 55: 207-225.

Arrese E.L. \& Wells M.A. 1994: Purification and properties of a phosphorylatable triacylglycerol lipase from the fat body of an insect, Manduca sexta. J. Lipid Res. 35: 1652-1659.

Arrese E.L. \& Wells M.A. 1997: Adipokinetic hormoneinduced lipolysis in the fat body of an insect, Manduca sexta: synthesis of sn-1,2-diacylglycerols. J. Lipid Res. 38: 68-76.

Arrese E.L., Rojas-Rivas B.I. \& Wells M.A. 1996: The use of decapitated insects to study lipid mobilization in adult Manduca sexta, effects of adipokinetic hormone and trehalose on fat body lipase activity. Insect Biochem. Mol. Biol. 26: $775-782$.
Beenakkers A.M.T. \& Scheres J.M.J.C. 1971: Dietary lipids and lipid composition of the fat body of Locusta migratoria. Insect Biochem. 1: 125-129.

BeEnakKers A.M.T., VAn der Horst D.J. \& VAN MarrewiJK W.J.A. 1985: Insect lipids and lipoproteins, and their role in physiological processes. Prog. Lipid Res. 24: 19-67.

Canavoso L.E. \& Wells M.A. 2000: Metabolic pathways for diacylglycerol biosynthesis and release in the midgut of larval Manduca sexta. Insect Biochem. Mol. Biol. 30: 1173-1180.

Canavoso L.E., Bertello L.E., De Lederkremer R.M. \& Rubiolo E.R. 1998: Effect of fasting on the composition of the fat body lipid of Dipetalogaster maximus, Triatoma infestans and Panstrongylus megistus (Hemiptera: Reduviidae). J. Comp. Physiol. (B) 168: 549-554.

Canavoso L.E., Jouni Z.E., Karnas K.J., Pennington J.E. \& Wells M.A. 2001: Fat metabolism in insects. Annu. Rev. Nutr. 21: 23-46.

Canavoso L.E., Frede S. \& Rubiolo E.R. 2004: Metabolic pathways for dietary lipids in the midgut of hematophagous Panstrongylus megistus (Hemiptera: Reduviidae). Insect Biochem. Mol. Biol. 34: 845-854.

Chino H. \& Downer R.G.H. 1982: Insect hemolymph lipophorin: a mechanism of lipid transport in insects. Adv. Biophys. 15: 67-92.

CvačKa J., Krafková E., Jiroš P. \& Valterová I. 2006: Computer-assisted interpretation of atmospheric pressure chemical ionisation mass spectra of triacylglycerols. Rapid Commun. Mass Spectrom. 20: 3586-3594.

Downer R.G.H. 1985: Lipid metabolism. In Kerkut G.A. \& Gilbert L.I. (eds): Comprehensive Insect Physiology, Biochemistry and Pharmacology. Vol. 10. Pergamon Press, Oxford, pp. 77-113.

Folch J., Lees M. \& Sloane-Stanley G.H. 1957: A simple method for the isolation and purification of total lipids from animal tissues. J. Biol. Chem. 226: 497-509.

GÄDE G. 2009: Peptides of the adipokinetic hormone/red pigment-concentrating hormone family: a new take on biodiversity. Ann. N. Y. Acad. Sci. 1163: 125-136.

GädE G. \& Goldsworthy G.J. 2003: Insect peptide hormones: a selective review of their physiology and potential application for pest control. Pest Manag. Sci. 59: 1063-1075.

Gäde G., Hoffmann K.H. \& Spring H. 1997: Hormonal regulation in insects: facts, gaps, and future directions. Physiol. Rev. 77: 963-1032.

GoldswORTHY G.J. 1994: The insect adipokinetic hormones: are they the insect glucagons? In Davey K.G., Peter R.E. \& Tobe S.S. (eds): Perspectives in Endocrinology: Proceedings of XII. International Congress of Comparative Endocrinology. National Research Council of Canada, Ottawa, pp. 486-492.

Hoffman A.G.D. \& Downer R.G.H. 1979: End product specificity of triacylglycerol lipases from intestine, fat body, muscle and haemolymph of the American cockroach, Periplaneta americana L. Lipids 14: 893-899.

Howard R.W. \& Stanley-Samuelson D.W. 1996: Fatty acid composition of fat body and Malpighian tubules of the tenebrionid beetle, Zophobas atratus: significance in eicosanoidmediated physiology. Comp. Biochem. Physiol. (B) 115: 429-437.

KEELEY L.L. 1985: Physiology and biochemistry of the fat body. In Kerkut G.A. \& Gilbert L.I. (eds): Comprehensive Insect Physiology, Biochemistry and Pharmacology. Vol. 3. Pergamon Press, Oxford, pp. 211-248.

KoDRík D. 2008: Adipokinetic hormone functions that are not associated with insect flight. Physiol. Entomol. 33: 171-180.

Kodrík D., Socha R., Šmek P., Zemek R. \& Goldsworthy G.J. 2000: A new member of the AKH/RPCH family that stimu- 
lates locomotory activity in the firebug, Pyrrhocoris apterus (Heteroptera). Insect Biochem. Mol. Biol. 30: 489-498.

Kodrík D., ŠIMEK P., LePŠA L. \& SochA R. 2002: Identification of the cockroach neuropeptide Pea-CAH-II as a second adipokinetic hormone in the firebug Pyrrhocoris apterus. Peptides 23: 585-587.

Kodrík D., Socha R. \& Syrová Z. 2003: Developmental and diel changes of adipokinetic hormone in CNS and haemolymph of the flightless wing-polymorphic bug, Pyrrhocoris apterus (L.). J. Insect Physiol. 49: 53-61.

Kodrík D., Socha R., Syrová Z. \& Zemek R. 2005: The effect of constant darkness on the content of adipokinetic hormone, adipokinetic response and walking activity in macropterous females of Pyrrhocoris apterus (L.). Physiol. Entomol. 30: $248-255$.

Kodrík D., Marco H.G., Šimek P., Socha R., ŠTys P. \& Gäde G. 2010: The adipokinetic hormones of Heteroptera: a comparative study. Physiol. Entomol. 35: 117-127.

KošŤÁL V. \& ŠIMEK P. 1998: Changes in fatty acid composition of phospholipids and triacylglycerols after cold-acclimation of an aestivating insect prepupa. J. Comp. Physiol. (B) $\mathbf{1 6 8}$ : 453-460.

MARTIN J.S. 1969a: Lipid composition of fat body and its contribution to the maturing oocytes in Pyrrhocoris apterus. $J$. Insect Physiol. 15: 1025-1045.

MARTIN J.S. 1969b: Studies on assimilation, mobilization, and transport of lipids by the fat body and haemolymph of Pyrrhocoris apterus. J. Insect Physiol. 15: 2319-2344.

Maxová A., Kodrík D., Zemek R. \& Socha R. 2001: Diel changes in adipokinetic response and walking activity of Pyrrhocoris apterus (L.) (Heteroptera) in relation to physiological status and wing dimorphism. Eur. J. Entomol. 98: 433-438.

MAYeR R.J. \& CANDY D.J. 1969: Control of haemolymph lipid concentration during locust flight: an adipokinetic hormone from the corpora cardiaca. J. Insect Physiol. 15: 611-620.

Mu H. \& Hoy C.E. 2000: Application of atmospheric pressure chemical ionization liquid chromatography - mass spectrometry in identification of lymph triacylglyceroles. J. Chromatogr. (B) 48: 425-437.

Murata M. \& Tojo S. 2002: Utilization of lipid for flight and reproduction in Spodoptera litura (Lepidoptera: Noctuidae). Eur. J. Entomol. 99: 221-224.

RANGAN V.S. \& Sмith S. 2002: Fatty acid synthesis in eukaryotes. In Vance D.E. \& Vance J.E. (eds): Biochemistry of Lipids, Lipoproteins and Membranes. Elsevier Science B.V., Paris, pp. 151-180.

SChNeIDER M. \& Dorn A. 1994: Lipid storage and mobilization by flight in relation to phase and age of Schistocerca gregaria females. Insect Biochem. Mol. Biol. 24: 883-889.

Socha R. 1993: Pyrrhocoris apterus (Heteroptera) - an experimental model species: a review. Eur. J. Entomol. 90: 241-286.

SochA R. \& KoDRíK D. 1999: Differences in adipokinetic response of Pyrrhocoris apterus (Heteroptera) in relation to wing dimorphism and diapause. Physiol. Entomol. 24: 278-284.

Socha R. \& Šula J. 1996: Differences in haemolymph proteins in relation to diapause and wing dimorphism in Pyrrhocoris apterus (L.) (Heteroptera: Pyrrhocoridae). J. Comp. Physiol. (B) 166: 382-387.

Socha R. \& ŠUla J. 2008: Regulation of the development of flight muscles in long-winged adults of the flightless bug, Pyrrhocoris apterus (Heteroptera: Pyrrhocoridae). Eur. J. Entomol. 105: 575-583.

SOCHA R. \& ZEMEK R. 2003: Wing morph-related differences in the walking pattern and dispersal in a flightless bug, Pyrrhocoris apterus (L.) (Heteroptera). Oikos 100: 35-43.

Socha R., Šula J. \& ZemeK R. 1997: Feeding, drinking and digestive enzyme activities in long- and short-day females of Pyrrhocoris apterus (Heteroptera). Physiol. Entomol. 22: 161-169.

Socha R., Šula. J. \& ZemeK R. 1998: Feeding behaviour, digestive physiology and lipid content in macropterous females of Pyrrhocoris apterus (L) (Heteroptera: Pyrrhocoridae). Physiol. Entomol. 23: 91-96.

Socha R., KodRíK D. \& ZemeK R. 1999: Adipokinetic hormone stimulates insect locomotor activity. Naturwissenschaften $\mathbf{8 8}$ : 85-86.

Socha R., Kodrík D., ŠimeK P. \& PatočKová M. 2004: The kind of AKH-mobilized energy substrates in insects can be predicted without a knowledge of the hormone structure. Eur. $J$. Entomol. 101: 29-35.

Socha R., Kodrík D. \& Šula J. 2005: Wing morph-specific differences in the metabolism and endocrine control of reserve mobilisation in adult males of a flightless bug, Pyrrhocoris apterus (L.) (Heteroptera). J. Comp. Physiol. (B) 175: 557-565.

TomČala A., Tollarová M., Overgaard J., Šimek P. \& KošŤál V. 2006: Seasonal acquisition of chill tolerance and restructuring of membrane glycerophospholipids in an overwintering insect: triggering by low temperature, desiccation and diapause progression. J. Exp. Biol. 209: 4102-4114.

TomČAla A., BÁrtů I., Š IMEK P. \& Kodrík D. 2010: Locust adipokinetic hormones mobilize diacylglycerols selectively. Comp. Biochem. Physiol. (B) 156: 26-32.

Turunen S. \& Crailsheim K. 1996: Lipid and sugar absorption. In Lehane M.J. \& Billingsley P.F. (eds): Biology of Insect Midgut. Chapman and Hall, London, pp. 293-320.

VAN DER HoRst D.J. 1982: Lipid transport in insects. In Mittler T.E. \& Dadd R.H. (eds): Metabolic Aspects of Lipid Nutrition in Insects. Westview Press, Boulder, CO, pp. 183-202.

VAN DER HORST D.J. \& RYAN R.O. 2005: Lipid transport. In Gilbert L.I., Iatrou K. \& Gill S.S. (eds): Comprehensive Molecular Insect Science. Vol. 4. Elsevier Pergamon, Kidlington, pp. 225-246.

Van Heusden M.C. \& Law J.H. 1989: An insect lipid transfer particle promotes lipid loading from fat body to lipoprotein. $J$. Biol. Chem. 264: 17287-17292. 